\title{
REMOÇÂO DO CHUMBO ATRAVÉS DA FIBRA DE COCO
}

\author{
Lucas Augusto Mendes de Morais ${ }^{1}$ \\ Mackaully Frederico Ferreira ${ }^{2}$ \\ Carlos Eduardo Sathler Ferreira ${ }^{3}$ \\ Larissa Siqueira Abadia ${ }^{4}$ \\ Cassia Cristina de Lima Simão Magalhães ${ }^{5}$
}

Resumo: Neste trabalho comprova-se a eficiência da adsorção da palha de coco, adsorvente alternativo e de baixo custo, na remoção de íons de $\mathbf{P b}^{2+}$ do meio aquoso, avaliando as possibilidades de aplicação desse método em futuros projetos. Um processo que tem alcançado grande importância nas últimas décadas é a adsorção no tratamento de efluentes. Será essa aplicação usada no tratamento de soluções de chumbo. Verificou-se também a sua eficiência. A remoção foi realizada no laboratório de Química do Colégio UNIVAP - Unidade Villa Branca e Instituto de Pesquisa e Desenvolvimento (IP\&D). Os resultados iniciais mostraram que a técnica de remoção de chumbo através da fibra de coco é muito eficiente e viável. Palavras-chave: Adsorção; Fibra de coco; Remoção; Chumbo; Meio ambiente.

\footnotetext{
1 Colégios UNIVAP - Unidade Villa Branca, Brasil. E-mail: lucasmmorais2010@hotmail.com.

2 Colégios UNIVAP - Unidade Villa Branca, Brasil. E-mail: mackaully.123@hotmail.com.

${ }^{3}$ Colégios UNIVAP - Unidade Villa , Brasil. E-mail: cadusathler@hotmail.com.

${ }^{4}$ Colégios UNIVAP - Unidade Villa Branca, Brasil. E-mail: larissa.siqueira_2010@hotmail.com.

${ }^{5}$ Colégios UNIVAP - Unidade Villa Branca, Brasil. E-mail: cassia@univap.br.
} 